\title{
Evidence for stable square ice from quantum Monte Carlo
}

\author{
Ji Chen, ${ }^{1,2,3}$ Andrea Zen, ${ }^{1,2,3}$ Jan Gerit Brandenburg, ${ }^{2,3,4}$ Dario Alfè, $, 1,2,3,5$ and Angelos Michaelides ${ }^{1,2,3, *}$ \\ ${ }^{1}$ Department of Physics and Astronomy, University College London, Gower Street, London WC1E 6BT, United Kingdom \\ ${ }^{2}$ London Centre for Nanotechnology, 17-19 Gordon Street, London WC1H OAH, United Kingdom \\ ${ }^{3}$ Thomas Young Centre, University College London, 20 Gordon Street, London WC1H OAJ, United Kingdom \\ ${ }^{4}$ Department of Chemistry, University College London, 20 Gordon Street, London WC1H OAH, United Kingdom \\ ${ }^{5}$ Department of Earth Sciences, University College London, Gower Street, London WC1E 6BT, United Kingdom
}

(Received 5 October 2016; published 6 December 2016)

\begin{abstract}
Recent experiments on ice formed by water under nanoconfinement provide evidence for a two-dimensional (2D) "square ice" phase. However, the interpretation of the experiments has been questioned and the stability of square ice has become a matter of debate. Partially this is because the simulation approaches employed so far (force fields and density functional theory) struggle to accurately describe the very small energy differences between the relevant phases. Here we report a study of 2D ice using an accurate wave-function based electronic structure approach, namely diffusion Monte Carlo (DMC). We find that at relatively high pressure, square ice is indeed the lowest enthalpy phase examined, supporting the initial experimental claim. Moreover, at lower pressures, a "pentagonal ice" phase (not yet observed experimentally) has the lowest enthalpy, and at ambient pressure, the "pentagonal ice" phase is degenerate with a "hexagonal ice" phase. Our DMC results also allow us to evaluate the accuracy of various density functional theory exchange-correlation functionals and force field models, and in doing so we extend the understanding of how such methodologies perform to challenging $2 \mathrm{D}$ structures presenting dangling hydrogen bonds.
\end{abstract}

DOI: 10.1103/PhysRevB.94.220102

Recent transmission electron microscopy (TEM) measurements and classical molecular dynamics simulations report that a new two-dimensional (2D) square phase of ice forms [1]. This phase is not part of the bulk ice phase diagram, but it was suggested that it is stabilized under confinement because of lateral pressure, estimated to be in the gigapascal (GPa), arising from the van der Waals (vdW) attraction between the graphene sheets. However, these experiments have been questioned [2], with it even being suggested that it is sodium chloride contamination and not ice that is responsible for the square symmetry observed. So far, it is not clear under what conditions (if any) square ice is stable.

Theoretical investigations of the stability of confined 2D ice at high lateral pressures can, in principle, help in disentangling this issue and in complementing experimental findings [3-10]. From a theoretical perspective, the prediction of square 2D ice can be traced back to Nagle's 1970's "unit model" of ice [3]. However, later atomistic force field (FF) simulations found that 2D ice prefers a buckled rhombic structure [4,5]. More recently, density functional theory (DFT) based investigations [6-9] have been performed. However, these have produced qualitatively different results depending on the precise details of the calculations and, in particular, on the choice of exchange-correlation (XC) functional. For instance, Chen et al. [7] found hexagonal and pentagonal structures to be stable phases at low pressures (Fig. 1). They also found that square ice is only stable in the GPa pressure

\footnotetext{
*angelos.michaelides@ucl.ac.uk
}

Published by the American Physical Society under the terms of the Creative Commons Attribution 4.0 International license. Further distribution of this work must maintain attribution to the author(s) and the published article's title, journal citation, and DOI. regime and that even at these pressures it is only favored enthalpicly by $<10 \mathrm{meV} / \mathrm{H}_{2} \mathrm{O}(1 / 4 \mathrm{kcal} / \mathrm{mol})$. Meanwhile, DFT results of Corsetti et al. [6] showed that the square ice structure is more stable than the hexagonal phase at all pressures. These disparate findings raise serious questions about the reliability of the adopted computational approaches (both DFT and FF) applied so far to 2D ice. Indeed, this is exemplary of a broader longstanding issue. The water and ice phase diagram is extremely challenging for any computational approach, because there can be competing phases within an energy range of only a few tens of $\mathrm{meV} / \mathrm{H}_{2} \mathrm{O}$ [11]. Achieving this accuracy is often beyond the capabilities of DFT XC functionals and most FF approaches [12,13]. Therefore, a study of $2 \mathrm{D}$ ice with a more accurate theoretical method is needed.

The development and application of electronic structure approaches with meV accuracy is now a thriving area of condensed matter research (see, e.g., Refs. [14-17]). Of the various methods available, diffusion Monte Carlo (DMC) is particularly attractive [18-24]. First, DMC has already been shown to offer the requisite accuracy for bulk ice phases by producing results in excellent agreement with experiment $[11,12,25,26]$. Second, owing to recent improvements in computational efficiency [27], it is now possible to obtain converged results on the large unit cells that must be considered when treating $2 \mathrm{D}$ ice with a many-body electronic structure approach. With this in mind, herein we report a DMC study of 2D ice. Our DMC calculations reveal that hexagonal and pentagonal phases are indeed the most stable 2D ice phases identified at low pressures. Perhaps of more interest, though, DMC calculations at $2 \mathrm{GPa}$ clearly support the existence of square ice at a high lateral pressure. As a further step we then use our DMC reference data to understand how the much more widely used DFT and FF approaches perform for such systems, so as to provide guidance for 


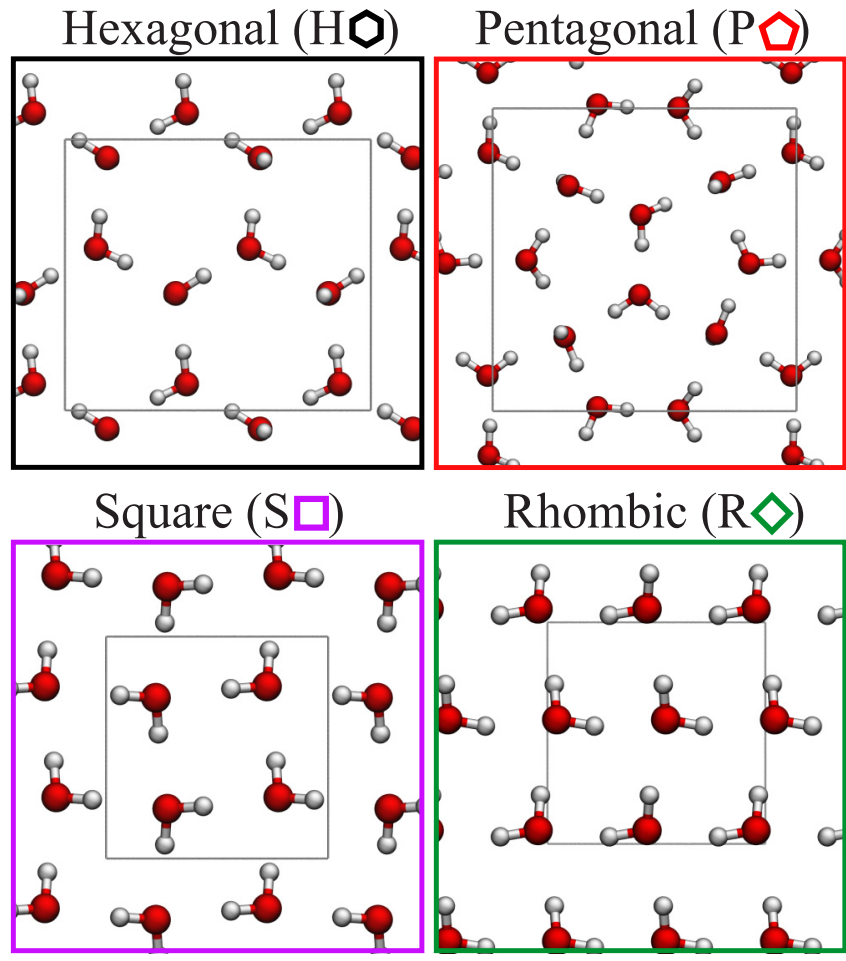

FIG. 1. The four most relevant $2 \mathrm{D}$ ice structures considered as part of this study: hexagonal $(\mathrm{H})$, pentagonal $(\mathrm{P})$, square $(\mathrm{S})$, and rhombic $(\mathrm{R})$. The gray boxes represent the primitive unit cells.

future studies on 2D ice and interfacial water. From this, several DFT XC functionals which perform well are identified and we also find that commonly used FFs such as extended simple point charge (SPC/E) [28] and four-point-transferable intermolecular potential (TIP4P) [29] tend to overstabilize high-density phases, helping to explain why such phases have been widely observed in FF studies.

Previous DFT studies suggest that four structureshexagonal, pentagonal, square, and rhombic (see Fig. 1) - are the most stable monolayer ice structures [7], so these are the focus of the current study. In the rhombic and square structures the water molecules are fourfold coordinated, as in bulk ice, but are arranged in the plane. The pentagonal and hexagonal structures have water molecules arranged similarly to a cut in bulk ice, so that there are some water molecules that are not fourfold coordinated and some with dangling hydrogen bonds. Specifically, in the hexagonal structure all water molecules are threefold coordinated and one-half of them have one dangling hydrogen bond; in the pentagonal structure, one-third of the water molecules have one dangling hydrogen bond and are fourfold coordinated, and the remaining molecules are threefold coordinated and have no dangling bonds. The CASINO code [30] has been used for the DMC calculations using Dirac-Fock pseudopotentials [31,32] with the locality approximation [33]. Slater-Jastrow trial wave functions with single Slater determinants were used and the single particle orbitals obtained from DFT-LDA (local density approximation) plane wave calculations reexpanded in terms of $B$ splines [34]. We used the DMC algorithm with the prescriptions of Zen et al. [27], which allows a DMC time step as large as
0.02 a.u. to be used with negligible time step errors. DMC calculations have been performed on structures optimized with DFT at a range of lateral pressures and under a uniform 2D confining potential fitted to DMC for values for the watergraphene interaction [35]. See Ref. [7] and the Supplemental Material therein for details of the confining potential used and discussions on the impact of using explicit graphene as the confining material. The structures used for DMC calculations have been obtained by performing geometry optimizations with the optimized Perdew-Burke-Ernzerhof-van der Waals (optPBE-vdW) functional [36]. Additional calculations have also been performed on selected structures obtained from the revised PBE-vdW (revPBE-vdW) functional [36], leading to similar results, as detailed in the Supplemental Material (SM) [37]. Further details of the setups used in the DMC, DFT, and FF calculations are also included in the SM.

In order to assess the stability of the different 2D ice structures, it is necessary to consider their enthalpy $(H)$ at $0 \mathrm{~K}$ [37]. The enthalpy is the sum of the binding energy $E_{\mathrm{b}}$ [38], the confinement energy $E_{\text {conf }}$, the pressure volume work $(P V)$, and the zero point energy (ZPE). To begin, we first discuss the accurate evaluation of $E_{\mathrm{b}}$ using DMC. In DMC, and other many-body methods, finite size (FS) errors can be sizable unless large supercells and/or correction terms are considered [39-41]. Since we are concerned with very small energy differences between the various phases, we have carefully addressed this issue with a series of calculations for increasing supercell size. Supercells with eight up to as many as 192 water molecules were considered, and for each system considered DMC simulations were run until the stochastic error of $E_{\mathrm{b}}$ was $\leqslant 3 \mathrm{meV} / \mathrm{H}_{2} \mathrm{O}$. The results obtained are plotted in Fig. 2 as a function of the inverse number of water molecules in the simulated supercell $1 / N_{w}$. From these calculations we have extrapolated $E_{\mathrm{b}}$ for the different structures to infinite system size. The extrapolated binding energies are summarized in Table I from where it can be seen that the hexagonal phase has the largest binding energy and the pentagonal phase is only marginally $\left(4 \mathrm{meV} / \mathrm{H}_{2} \mathrm{O}\right)$ less stable. These calculations also reveal that the FS error leads to an overestimate of $E_{\mathrm{b}}$ by more than $10 \mathrm{meV} / \mathrm{H}_{2} \mathrm{O}$ in the smallest cells $\left(N_{w}<20\right)$, and the relative energies between the different structures are fairly insensitive to the size of the cell used. We note that the $N_{w} \rightarrow \infty$ extrapolations are obtained by assuming that FS errors are proportional to $1 / N_{w}$. In the SM we show that other choices do not alter the extrapolated values of $E_{\mathrm{b}}$ [37].

With the binding energies obtained, the enthalpies can be further calculated considering $E_{\text {conf }}, P V$, and ZPE. Each of these additional terms has been obtained with DFT, and upon putting everything together, we find that at vanishing pressure the pentagonal and hexagonal structures have essentially the same enthalpy. The values obtained come within $1 \mathrm{meV} / \mathrm{H}_{2} \mathrm{O}$ of each other, which is within the stochastic errors of our DMC simulations $\left(3 \mathrm{meV} / \mathrm{H}_{2} \mathrm{O}\right)$. Although it is hard to differentiate the pentagonal and the hexagonal structures at vanishing pressure, based on the enthalpies obtained we would expect the higher-density pentagonal phase to become more stable than the hexagonal phase at small applied pressure, as first proposed using DFT [7]. The square and rhombic structures are 13 and $42 \mathrm{meV} / \mathrm{H}_{2} \mathrm{O}$ higher in enthalpy, respectively, than 


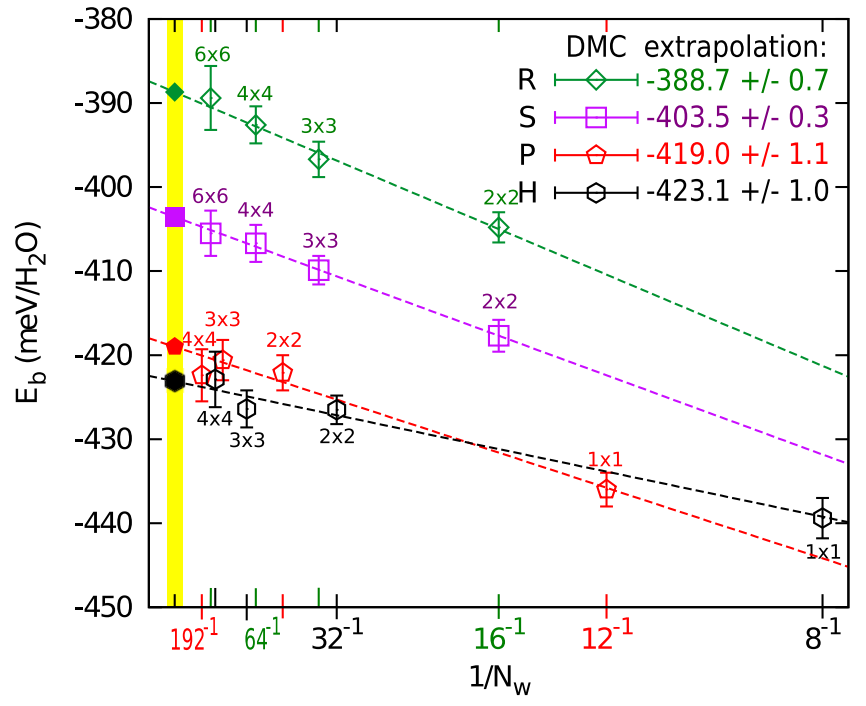

FIG. 2. DMC binding energies $E_{\mathrm{b}}$ for the zero pressure structures of free-standing $2 \mathrm{D}$ ice in the hexagonal $(\mathrm{H})$, pentagonal $(\mathrm{P})$, square (S), and rhombic (R) structures (see Fig. 1) as a function of the inverse number of water molecules in the simulation cell $1 / N_{w}$. Open symbols correspond to DMC evaluations of $E_{\mathrm{b}}$, and error bars are one standard deviation. For each point we indicate the supercell size in terms of the primitive unit cell. The solid symbols on the yellow region are the extrapolated values $E_{\infty}$ from a fit with the function $E\left(N_{w}\right)=E_{\infty}-c / N_{w}$; values and errors of the fit are reported on the top right corner.

the pentagonal structure at zero pressure. Note that the rhombic structure is buckled, thus also the confining energy penalizes it compared to the other structures.

In the 2D ice phase diagram, the question of the stability of square ice is particularly interesting because it is arguably the only experimentally observed 2D ice, and disagreements exist between FFs and DFT, and also within DFT itself [1,2,6,7]. We have already seen that the square phase is less stable than the pentagonal and hexagonal phases at low pressures. However, it has been estimated that the pressure in graphene nanocapillaries can be as large as several GPa due to the van der Waals forces pulling the graphene sheets together [1]. Therefore, we carried out DMC calculations on structures at $2 \mathrm{GPa}$. In Table I we report results for pentagonal, square, and rhombic structures; the hexagonal structure becomes unstable at $2 \mathrm{GPa}$. We find that the square structure is indeed the most stable. Its enthalpy is lower than the pentagonal structure by $28 \mathrm{meV} / \mathrm{H}_{2} \mathrm{O}$ and lower than the rhombic structure by $17 \mathrm{meV} / \mathrm{H}_{2} \mathrm{O}$. Although this study has focused on relative enthalpies at $0 \mathrm{~K}$, we have also estimated the relative free energies of the various phases by taking into account the vibrational contributions to the free energies. As shown in the SM (Table SII) at $300 \mathrm{~K}$ the square phase remains the most stable phase at $2 \mathrm{GPa}$.

DMC has helped to clarify the relative stabilities of the various $2 \mathrm{D}$ ices at ambient and high pressure. We now use these DMC benchmarks to understand how various DFT XC functionals and FF models perform on such structures. This is important to establish as DFT and FFs are widely used to examine 2D ice, interfacial, and confined water. Here we discuss
TABLE I. Properties of the various 2D ice structures at zero pressure and $2 \mathrm{GPa}$. The values reported are the binding energy $E_{\mathrm{b}}\left(\mathrm{meV} / \mathrm{H}_{2} \mathrm{O}\right)$ obtained from DMC, the confinement energy $E_{\text {conf }}$ $\left(\mathrm{meV} / \mathrm{H}_{2} \mathrm{O}\right)$, the lateral area per water $A\left(\AA^{2} / \mathrm{H}_{2} \mathrm{O}\right)$, the pressure volume work $P V=P \times A \times w\left(\mathrm{meV} / \mathrm{H}_{2} \mathrm{O} ; w\right.$ is the width of the confinement), the zero point energy $(\mathrm{ZPE})\left(\mathrm{meV} / \mathrm{H}_{2} \mathrm{O}\right)$, and the enthalpy $H$ (inclusive of ZPE). Also reported is the enthalpy difference with respect to the most stable structure: $\Delta_{\text {pen }}=(H)-$ $(H)$ [pentagonal] at zero pressure, $\Delta_{\mathrm{sq}}=(H)-(H)$ [square] at $2 \mathrm{GPa}$. All the DMC stochastic errors associated with $E_{\mathrm{b}}$ are $\leqslant 3 \mathrm{meV} / \mathrm{H}_{2} \mathrm{O}$. $E_{\text {conf }}, A, P V$, and ZPE were obtained using DFT with the optPBE$\mathrm{vdW}$ functional. See SM for additional details.

\begin{tabular}{lccccccc}
\hline \hline Pressure & $E_{\mathrm{b}}$ & $E_{\text {conf }}$ & $A$ & $P V$ & ZPE & $\mathrm{H}$ & \\
\hline 0 & & & & & & & $\Delta_{\text {pen }}$ \\
Hexagonal & -423 & 21 & 9.728 & 0 & 676 & 274 & 1 \\
Pentagonal & -419 & 20 & 8.635 & 0 & 672 & 273 & 0 \\
Square & -404 & 18 & 7.974 & 0 & 672 & 286 & 13 \\
Rhombic & -389 & 37 & 7.999 & 0 & 667 & 315 & 42 \\
\hline 2 GPa & & & & & & & $\Delta_{\text {sq }}$ \\
Pentagonal & -380 & 52 & 7.710 & 577 & 674 & 923 & 28 \\
Square & -374 & 47 & 7.193 & 539 & 683 & 895 & 0 \\
Rhombic & -385 & 99 & 6.905 & 517 & 681 & 912 & 17 \\
\hline \hline
\end{tabular}

the main indications that come out of these comparisons. In Fig. 3 (left panel) we plot the average error in the binding energy of 2D ice structures as obtained from a variety of approaches. From this we find that several XC functionals,

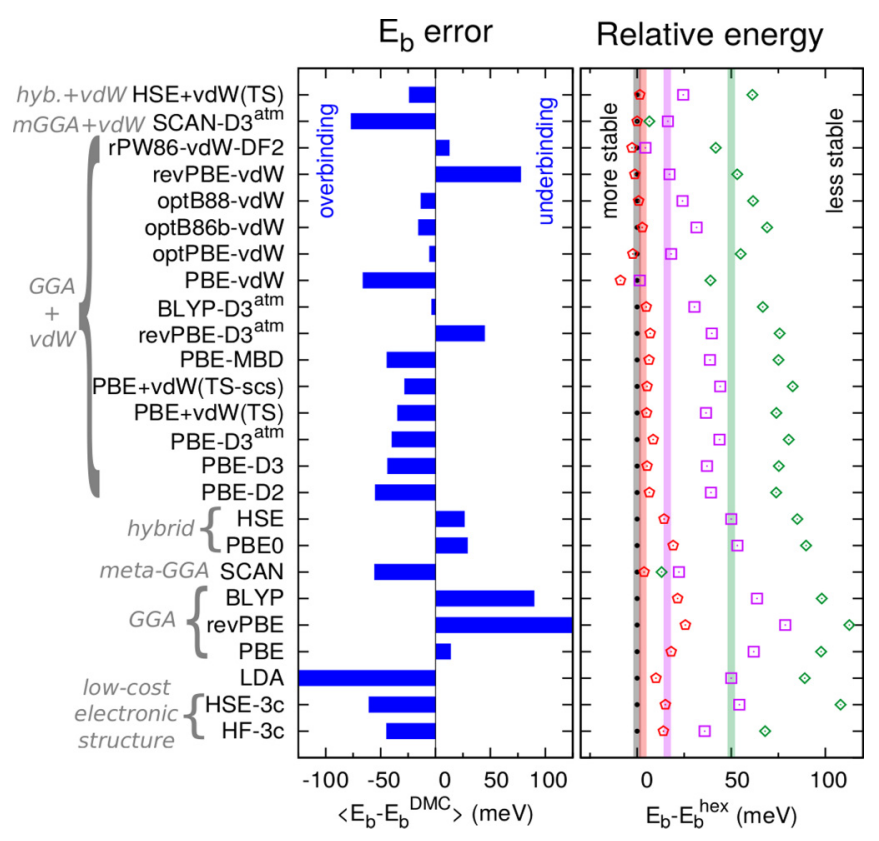

FIG. 3. The left panel reports the average error (blue bars) for the $E_{b}$ evaluations, taking DMC as the reference, using a selection of XC functionals. See the SM for information on each method. The right panel reports the energy difference $E_{\mathrm{b}}-E_{\mathrm{b}}^{\text {hex }}$ at zero pressure for each of the considered methods. The benchmark DMC evaluations are shown as color-coded bands, the widths of which represent the stochastic error (i.e., $\pm \sigma$ ). Color and symbol conventions are the same as in the previous figures. Results are on optPBE-vdW optimized structures at zero pressure. Similar plots for relaxed structures and for $2 \mathrm{GPa}$ are reported in the SM. 


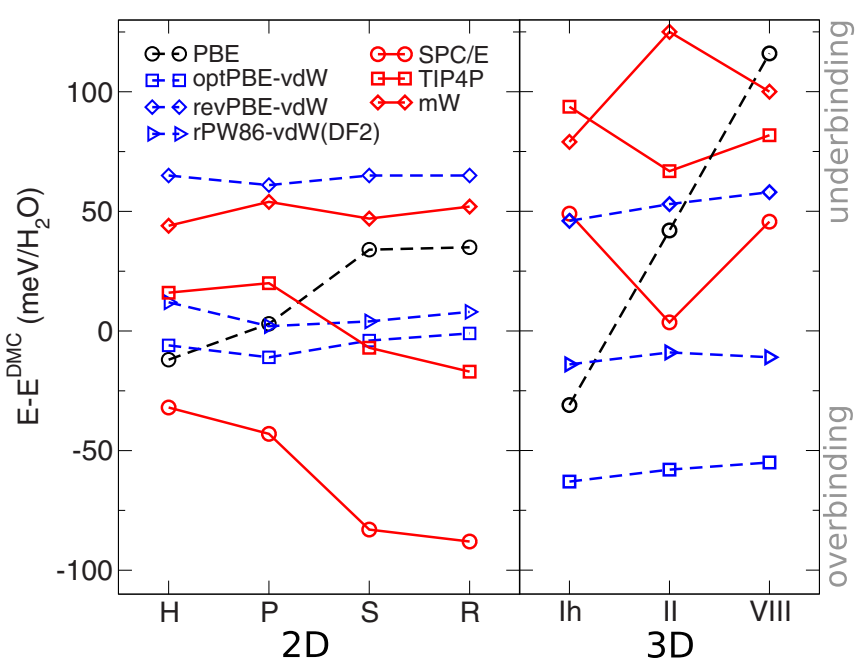

FIG. 4. Binding energy difference $E-E^{\mathrm{DMC}}$ with respect to DMC for various DFT XC functionals and FF models for the 2D and some bulk (3D) ice structures at zero pressure. For clarity, only a subset of the FF potentials and DFT XC functionals are plotted here; data with other approaches are included in the SM. Bulk ice results are taken from Refs. [11,25] (DMC and DFT) and Ref. [42] (FF). In both panels the densities of the phases increase from left to right.

namely, HSE-vdW(TS), rPW86-vdW-DF2, optB88-vdW, optB86b-vdW, optPBE-vdW, BLYP-D3 ${ }^{\text {atm }}$, and PBE perform well, yielding an average error of $<25 \mathrm{meV} / \mathrm{H}_{2} \mathrm{O}$. Apart from binding energies averaged over the four structures, it is also crucial to predict correct relative energies. As shown by the relative energies of the four 2D ice structures (Fig. 3 right), we find that only the vdW inclusive approaches mentioned above provide satisfactory predictions. The long-range part of the $\mathrm{vdW}$ force therefore plays an important role in 2D ice. Interestingly, SCAN, a recently developed meta-GGA (generalized gradient approximation) functional that partially accounts for the medium-range vdW force [43,44], does not perform particularly well for these systems and overbinds all phases.

Extending the analysis, in Fig. 4 we plot the energy error of several XC functionals and FFs for both 2D and 3D ice. Results are shown in order of increasing density for both 2D and 3D ice. On comparing 2D and 3D ice we find that while vdW is important in 2D it plays a smaller role than it is known to play in $3 \mathrm{D}[11,25]$. This can be seen, for example, by comparing the slopes of the PBE curves in 2D and $3 \mathrm{D}$, where it can be seen that the PBE curve in 3D is much steeper. We also show in the SM that the reduced significance of $\mathrm{vdW}$ is due to the lower coordination of water molecules in 2D ice than in 3D. The difference of the vdW contribution in 2D and 3D leads to a contrasting performance of some of the $\mathrm{vdW}$ functionals for $2 \mathrm{D}$ and $3 \mathrm{D}$ ice, as shown in Fig. 4. Overall, we find that the rPW86-vdW-DF2 functional yields the smallest errors for both $2 \mathrm{D}$ and 3D ice structures, and represents a good choice for future studies of these and related systems.

In Fig. 4 we also present results with several FF models which have been widely used to study confined water and ice $[1,4,5,45,46]$. Our calculations show that the SPC/E and TIP4P models overestimate the binding energy of the high-density $2 \mathrm{D}$ ice structures more than the low-density ones. The difference between the errors on the hexagonal and rhombic structures is about $40 \mathrm{meV} / \mathrm{H}_{2} \mathrm{O}$ for TIP4P and about $60 \mathrm{meV} / \mathrm{H}_{2} \mathrm{O}$ for SPC/E. This error comes from both the Coulomb and the van der Waals components of the potential (Fig. S6). Results of TIP4P/2005 [47] and TIP4P/Ice [48] are also reported in the SM and typically they show a consistent shift of energy for all structures compared with TIP4P. Therefore, our results help to explain why rhombic and square structures are often seen in 2D ice simulations using these models. We have also performed calculations using the $\mathrm{mW}$ model [49], a widely used coarse-grained model of water. Although it significantly underestimates the binding energies of the $2 \mathrm{D}$ ice structures by approximately $50 \mathrm{meV} / \mathrm{H}_{2} \mathrm{O}$, it does very well in reproducing the relative energies of $2 \mathrm{D}$ ice. This good performance on the relative energies is also consistent with the fact that a pentagonal ice structure has been observed in $\mathrm{mW}$ simulations of confined water [46].

To conclude, our large-scale DMC simulations of 2D ice reveal that at ambient pressure the most stable structures are hexagonal and pentagonal phases. In addition, our calculations show that at high pressure, 2D square ice is more stable at $0 \mathrm{~K}$ than the other phases considered. The data and insight obtained here are important for current understanding of confined 2D ice in general and are also relevant to the TEM measurements of Algara-Siller et al. [1]. We note that, although our calculations find that square ice is stable, they do not rule out the possibility, as suggested by Zhou et al., that sodium chloride could have been observed in the initial measurements [2]. The influence of finite temperature, growth kinetics, and the finite size of the particles that form have also yet to be investigated in detail. Clearly more work is needed from experiment and theory in order to substantiate the existence of square ice, and to potentially observe the predicted pentagonal ice phase. Finally, from a theoretical point of view, our study reveals the different performance of many widely used DFT functionals and FF models on 2D ice. We find that the role of $\mathrm{vdW}$ forces in 2D and 3D ice is different, thus it is important to consider both 2D and 3D ice in order to reach a consistent picture of $\mathrm{vdW}$ inclusive XC functionals. We also rationalize observations in previous FF studies by showing that widely used FF models incorrectly stabilize the high-density $2 \mathrm{D}$ ice phases.

J.C., A.Z., and A.M. are supported by the European Research Council under the European Union's Seventh Framework Programme (FP/2007-2013)/ERC Grant Agreement No. 616121 (HeteroIce project). A.Z. and A.M.'s work is also sponsored by the Air Force Office of Scientific Research, Air Force Material Command, USAF, under Grant No. FA8655-12-1-2099. A.M. is also supported by the Royal Society through a Royal Society Wolfson Research Merit Award. J.G.B acknowledges support by the Alexander von Humboldt foundation within the Feodor-Lynen program. We are also grateful for computational resources to ARCHER, UKCP consortium (EP/F036884/1), the London Centre for Nanotechnology, UCL Research Computing, and Oak Ridge Leadership Computing Facility (No. DE-AC05-00OR22725).

J.C. and A.Z. contributed equally to this work. 
[1] G. Algara-Siller, O. Lehtinen, F. C. Wang, R. R. Nair, U. Kaiser, H. A. Wu, A. K. Geim, and I. V. Grigorieva, Nature (London) 519, 443 (2015).

[2] W. Zhou, K. Yin, C. Wang, Y. Zhang, T. Xu, A. Borisevich, L. Sun, J. C. Idrobo, M. F. Chisholm, S. T. Pantelides, R. F. Klie, and A. R. Lupini, Nature (London) 528, E1 (2015).

[3] J. Nagle, Chem. Phys. 43, 317 (1979).

[4] R. Zangi and A. E. Mark, Phys. Rev. Lett. 91, 025502 (2003).

[5] K. Koga and H. Tanaka, J. Chem. Phys. 122, 104711 (2005).

[6] F. Corsetti, P. Matthews, and E. Artacho, Sci. Rep. 6, 18651 (2016).

[7] J. Chen, G. Schusteritsch, C. J. Pickard, C. G. Salzmann, and A. Michaelides, Phys. Rev. Lett. 116, 025501 (2016).

[8] F. Corsetti, J. Zubeltzu, and E. Artacho, Phys. Rev. Lett. 116, 085901 (2016).

[9] T. Roman and A. Groß, J. Phys. Chem. C 120, 13649 (2016).

[10] M. Sobrino Fernandez Mario, M. Neek-Amal, and F. M. Peeters, Phys. Rev. B 92, 245428 (2015).

[11] B. Santra, J. Klimeš, D. Alfè, A. Tkatchenko, B. Slater, A. Michaelides, R. Car, and M. Scheffler, Phys. Rev. Lett. 107, 185701 (2011).

[12] M. J. Gillan, D. Alfè, and A. Michaelides, J. Chem. Phys. 144, 130901 (2016).

[13] G. A. Cisneros, K. T. Wikfeldt, L. Ojamäe, J. Lu, Y. Xu, H. Torabifard, A. P. Bartk, G. Csányi, V. Molinero, and F. Paesani, Chem. Rev. 116, 7501 (2016).

[14] G. H. Booth, A. Grüneis, G. Kresse, and A. Alavi, Nature (London) 493, 365 (2013).

[15] L. Schimka, J. Harl, A. Stroppa, A. Grüneis, M. Marsman, F. Mittendorfer, and G. Kresse, Nat. Mater. 9, 741 (2010).

[16] A. Michaelides, T. J. Martinez, A. Alavi, G. Kresse, and F. R. Manby, J. Chem. Phys. 143, 102601 (2015).

[17] J. Yang, W. Hu, D. Usvyat, D. Matthews, M. Schütz, and G. K.-L. Chan, Science 345, 640 (2014).

[18] W. M. C. Foulkes, L. Mitas, R. J. Needs, and G. Rajagopal, Rev. Mod. Phys. 73, 33 (2001).

[19] L. K. Wagner and P. Abbamonte, Phys. Rev. B 90, 125129 (2014).

[20] M. A. Morales, J. R. Gergely, J. McMinis, J. M. McMahon, J. Kim, and D. M. Ceperley, J. Chem. Theory Comput. 10, 2355 (2014).

[21] A. Benali, L. Shulenburger, N. A. Romero, J. Kim, and O. A. von Lilienfeld, J. Chem. Theory Comput. 10, 3417 (2014).

[22] G. Mazzola and S. Sorella, Phys. Rev. Lett. 114, 105701 (2015).

[23] A. Zen, Y. Luo, G. Mazzola, L. Guidoni, and S. Sorella, J. Chem. Phys. 142, 144111 (2015).

[24] M. Dubecký, L. Mitas, and P. Jurečka, Chem. Rev. 116, 5188 (2016).

[25] B. Santra, J. Klimeš, A. Tkatchenko, D. Alfè, B. Slater, A. Michaelides, R. Car, and M. Scheffler, J. Chem. Phys. 139, 154702 (2013).
[26] D. Quigley, D. Alfè, and B. Slater, J. Chem. Phys. 141, 161102 (2014).

[27] A. Zen, S. Sorella, M. J. Gillan, A. Michaelides, and D. Alfè, Phys. Rev. B 93, 241118(R) (2016).

[28] H. J. C. Berendsen, J. R. Grigera, and T. P. Straatsma, J. Phys. Chem. 91, 6269 (1987).

[29] W. L. Jorgensen, J. Chandrasekhar, J. D. Madura, R. W. Impey, and M. L. Klein, J. Chem. Phys. 79, 926 (1983).

[30] R. J. Needs, M. D. Towler, N. D. Drummond, and P. L. Rios, J. Phys.: Condens. Matter 22, 023201 (2010).

[31] J. R. Trail and R. J. Needs, J. Chem. Phys. 122, 014112 (2005).

[32] J. R. Trail and R. J. Needs, J. Chem. Phys. 122, 174109 (2005).

[33] L. Mitas, E. L. Shirley, and D. M. Ceperley, J. Chem. Phys. 95 , 3467 (1991).

[34] D. Alfè and M. J. Gillan, Phys. Rev. B 70, 161101 (2004).

[35] J. Ma, A. Michaelides, D. Alfè, L. Schimka, G. Kresse, and E. Wang, Phys. Rev. B 84, 033402 (2011).

[36] J. Klimeš, D. R. Bowler, and A. Michaelides, J. Phys.: Condens. Matter 22, 022201 (2010).

[37] See Supplemental Material at http://link.aps.org/supplemental/ 10.1103/PhysRevB.94.220102 for computational details, extended data, additional notes, and structural files.

[38] $E_{\mathrm{b}}$ is calculated as the difference between the energy per water in the free-standing 2D ice sheet minus the energy of an isolated water molecule (see SM).

[39] C. Lin, F. H. Zong, and D. M. Ceperley, Phys. Rev. E 64, 016702 (2001).

[40] S. Chiesa, D. M. Ceperley, R. M. Martin, and M. Holzmann, Phys. Rev. Lett. 97, 076404 (2006).

[41] H. Kwee, S. Zhang, and H. Krakauer, Phys. Rev. Lett. 100, 126404 (2008).

[42] E. Sanz, C. Vega, J. L. F. Abascal, and L. G. MacDowell, Phys. Rev. Lett. 92, 255701 (2004).

[43] J. Sun, R. C. Remsing, Y. Zhang, Z. Sun, A. Ruzsinszky, H. Peng, Z. Yang, A. Paul, U. Waghmare, X. Wu, M. L. Klein, and J. P. Perdew, Nat. Chem. 8, 831 (2016).

[44] J. G. Brandenburg, J. E. Bates, J. Sun, and J. P. Perdew, Phys. Rev. B 94, 115144 (2016).

[45] K. Koga, H. Tanaka, and X. C. Zeng, Nature (London) 408, 564 (2000).

[46] J. C. Johnston, N. Kastelowitz, and V. Molinero, J. Chem. Phys. 133, 154516 (2010).

[47] J. L. F. Abascal and C. Vega, J. Chem. Phys. 123, 234505 (2005).

[48] J. L. F. Abascal, E. Sanz, R. García Fernández, and C. Vega, J. Chem. Phys. 122, 234511 (2005).

[49] V. Molinero and E. B. Moore, J. Phys. Chem. B 113, 4008 (2009). 